See Article page XXX.

\section{Commentary: Many things remain unlearned and undetermined}

\author{
Takashi Nitta, MD, PhD
}

Permanent pacemaker (PM) implantation is a major complication after the maze procedure. Although an implanted PM enables 24-hour monitoring of cardiac rhythm with intracardiac electrograms and allows continuous and objective understanding of the electrophysiologic condition of patients after the maze procedure, there are potential risks, such as infection, venous thrombosis, and occlusion, PM-mediated tachyarrhythmias, and others. Although the incidence of permanent PM implantation after the maze procedure has been reported as being from $2.5 \%$ to $21.5 \%$, Kakuta and colleagues ${ }^{1}$ reported $1.6 \%$ and $7.3 \%$ incidences of in-hospital and late-onset PM implantation after the modified maze procedure, respectively. The mortality and morbidities were not significantly different between the patients with or without a PM. It should be meaningful to review the mechanism of bradycardia after the maze procedure and discuss the factors for the postoperative PM implantation.

There are various factors that can influence the incidence of PM implantation after a surgery for atrial fibrillation and the factors may be classified as intraoperative (surgical) or nonsurgical. Mechanical or thermal injuries to the cardiac pulse generating or conduction system, such as the sinoatrial node (SAN), intra-atrial conduction tracts, and atrioventricular node, and interruption of the blood flow to the above system are the most likely intraoperative reasons for postoperative bradycardia and in-hospital PM implantation. The SAN pacemaker cells widely distribute along the crista terminalis and form a complex. ${ }^{2}$ The activations sprouting from the SAN are complex, and changing conduction out of the node-coupled with extranodal pacemakers-combines with the autonomic nervous system to

\footnotetext{
From the Department of Cardiovascular Surgery, Nippon Medical School, Tokyo, Japan.

Disclosures: The author reported no conflicts of interest.

The Journal policy requires editors and reviewers to disclose conflicts of interest and to decline handling or reviewing manuscripts for which they may have a conflict of interest. The editors and reviewers of this article have no conflicts of interest.

Received for publication Oct 15, 2021; revisions received Oct 15, 2021; accepted for publication Oct 18, 2021.

Address for reprints: Takashi Nitta, MD, PhD, Nippon Medical School, 1-1-5

Sendagi, Bunkyo-ku, Tokyo, Japan 113-8602 (E-mail: nitta@nms.ac.jp).

J Thorac Cardiovasc Surg 2021; $\mathbf{\square}: 1-2$

$0022-5223 / \$ 36.00$

Copyright (c) 2021 by The American Association for Thoracic Surgery

https://doi.org/10.1016/j.jtcvs.2021.10.027
}

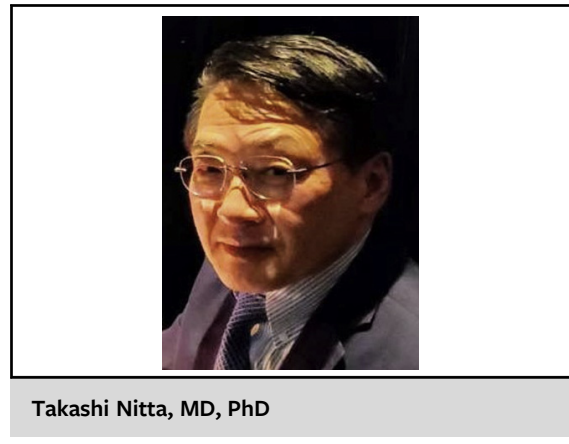

CENTRAL MESSAGE

Pacemaker implantation is a wellestablished therapy for bradycardia after the maze procedure with satisfactory outcomes, but many things remain unlearned or undetermined.

control heart rate and the pattern of impulse origin within the atria. ${ }^{3}$ Surgeons should pay attention not only to a small region of SAN, but also a wider region around the SAN to avoid any injuries to the widely distributed pacemaker complex. As the authors mention, the anatomical variance of the SAN artery is higher than most surgeons think. ${ }^{4}$ The atrial branches, particularly to the SAN, should be checked by the coronary angiograms before performing the maze procedure.

The authors modified the maze procedure by deleting the superior vena cava (SVC) ablation line. Avoidance of SAN injuries by deleting the SVC line might be the reason for the low incidence of PM implantation after the procedure. Among the fundamental principles of the maze procedure is prevention of any potential reentrant activations circulating around the anatomical obstacles, such as the SVC. ${ }^{5}$ Theoretically, deletion of the SVC ablation line increases the risk of postoperative reentrant tachycardia around the SVC. However, if the incidence of postoperative reentrant atrial tachycardia around the SVC is negligibly low; that is, validated by an objective controlled study, this modification of the maze procedure may be useful in avoidance of postoperative PM implantation.

There have been so many modified maze procedures performed. Inappropriate lesion sets of the modified maze procedures have a risk of injuring the SAN or other pulse-generating system, or interrupting the blood flow to the significant region and disturb a physiological 
conduction of the atrial activation. For example, the left atrial incision of the superior transseptal approach absolutely divides the SAN artery in normal hearts and possibly divides the aberrant SAN artery originating from the left circumflex artery. ${ }^{4}$ Even if the SAN survives the ischemia, the activation from the SAN travels a long path, detouring around the superior left atrial incision, to reach the atrioventricular node.

The nonsurgical factors for in-hospital or late-onset PM implantation after the maze procedure include preexisting dysfunction of the SAN or postoperative progressive dysfunction of the SAN, and other electrophysiologic abnormalities of intra-atrial conduction or atrioventricular node. The authors determined preoperative duration of atrial fibrillation and age as the significant factors for late-onset PM implantation after the modified maze procedure. ${ }^{1}$ In addition, the patients with long-standing persistent atrial fibrillation had a higher incidence of late-onset PM implantation compared with those with other types of atrial fibrillation. Perpetuation of rapid atrial activation progressively reduces the densities of transient outward current and L-type calcium ion current, resulting in shortening of the refractory period. ${ }^{6}$ This is called electrical remodeling, and the mechanism why the long-standing persistent atrial fibrillation is refractory to pharmacological and nonpharmacological therapies. ${ }^{7,8}$ Persistent rapid atrial activation also results in SAN dysfunction evidenced by prolonged SAN recovery time and slower intrinsic hearts. ${ }^{9}$ These changes are usually reversed gradually after termination of atrial fibrillation. However, some patients resist the reverse remodeling or even progress the electrical remodeling. Fibrosis of atrial tissue has been shown to be associated with the refractory reverse remodeling. ${ }^{10,11}$ Aging is another factor for late-onset PM implantation. The SAN pacemaker cells are interspersed with interstitial collagen. ${ }^{12}$ The collagen content increases with advancing age and may affect the propagation of the activation from the SAN pacemaker cells to the surrounding tissue. Increased right atrial pressure or volume stretches the SAN region may alter the atrial electrophysiology, and results in SAN dysfunction. In the present study, the frequency of PM implantation was not significantly high in the patients with severe tricuspid regurgitation and was rather low in those with dilated left atrium. This is probably because the patients with dilated right atrium or left atrium were not indicated for the maze procedure and excluded from the analysis.
Surgeons should pay more attention to the mechanical and thermal injuries to the SAN and other atrial structures that are essential for the physiological atrial activation, and to the normal and aberrant atrial coronary arteries. The lesion sets of the original maze procedure and the following modifications were designed based on the depth of understanding of the anatomy and physiology of the heart, followed by careful validation in animal studies. ${ }^{5}$ Any modification of the lesion set should be thoroughly considered and examined before been applied in patients. The mechanism for postoperative progression of electrical remodeling should be further studied to innovate pharmacological or nonpharmacological therapies to halt the remodeling progress. These studies would decrease the frequency of late-onset PM implantation and benefit patients after the maze procedure.

\section{References}

1. Kakuta, et al. Incidence of and risk factors for pacemaker implantation after the modified cryo-maze procedure for atrial fibrillation. J Thorac Cardiovasc Surg. 2022. XXX:XXX.

2. Boineau JP, Canavan TE, Schuessler RB, Cain ME, Corr PB, Cox JL. Demonstration of a widely distributed atrial pacemaker complex in the human heart. Circulation. 1988;77:1221-37.

3. Schuessler RB, Boineau JP, Bromberg BI. Origin of the sinus impulse. J Cardiovasc Electrophysiol. 1996;7:263-74.

4. Vikse J, Henry BM, Roy J, Ramakrishnan PK, Hsieh WC, Walocha JA, et al. Anatomical variations in the sinoatrial nodal artery: a meta-analysis and clinical considerations. Plos One. 2016;11:e0148331.

5. Cox JL, Schuessler RB, D’Agostino HJ, Stone CM, Chang BC, Cain ME, et al. The surgical treatment of atrial fibrillation. III. Development of a definitive surgical procedure. J Thorac Cardiovasc Surg. 1991;101:569-83.

6. Yue L, Feng J, Gaspo R, Li GR, Wang Z, Nattel S. Ionic remodeling underlying action potential changes in a canine model of atrial fibrillation. Circ Res. 1997; 81:512-25.

7. Wijffels MCEF, Kirchhof CJHJ, Dorland R, Allessie MA. Atrial fibrillation begets atrial fibrillation. Circulation. 1995;92:1954-68.

8. Goette A, Honeycutt C, Langberg JJ. Electrical remodeling in atrial fibrillation. Time course and mechanisms. Circulation. 1996;94:2968-74.

9. Elvan A, Wylie K, Zipes DP. Pacing-induced chronic atrial fibrillation impairs sinus node function in dogs. Electrophysiological remodeling. Circulation. 1996;94:2953-60

10. Oakes RS, Badger TJ, Kholmovski EG, Akoum N, Burgon NS, Fish EN, et al. Detection and quantification of left atrial structural remodeling with delayed-enhancement magnetic resonance imaging in patients with atrial fibrillation. Circulation. 2009;119:1758-67.

11. Marrouche NF, Wilber D, Hindricks G, Jais P, Akoum N, Marchlinski F, et al. Association of atrial tissue fibrosis identified by delayed enhancement MRI and atrial fibrillation catheter ablation: the DECAAF study. JAMA. 2014;311: 498-506.

12. Alings AM, Abbas RF, Bouman LN. Age-related changes in structure and relative collagen content of the human and feline sinoatrial node. A comparative study. Eur Heart J. 1995;16:1655-67. 